\title{
Improved Particle Swarm Optimization for Designing Tobacco Logistics Scheme
}

\author{
Zhao Li \\ Department of business \\ Shaanxi institute of international trade \& commerce \\ Xian Yang, China \\ zhaoli0108@163.com
}

\author{
Chen Yong \\ Department of Automatic \\ Air Force Engineering University \\ Xi'an, China \\ cheny_043@163.com
}

\begin{abstract}
With the minimal delivery cost as the managing target for the tobacco enterprise, the common mathematic model of tobacco logistics is built and the optimal solution is given in this paper. The main problem in tobacco logistics is to choose an effective vehicle routing, which can be solved by particle swarm optimization when the number of tobacco traders is small enough. But with the increasing of tobacco traders, the vehicle routing scheme is becoming more complicated. By introducing the cross-over operation and the mutation operation to the particles, the optimization capability is improved remarkably to achieve the best tobacco delivery method. The example shows that the optimal tobacco delivery routes can be obtained by the improved particle swarm optimization effectively comparing with genetic algorithms.
\end{abstract}

Keywords-tobacco logistics; vehicle routing problem; combined nonlinear optimization; particle swarm optimization

\section{INTRODUCTION}

Tobacco logistics, characterized by excessive delivery points and complicated delivery routes, is a typical combined Nondeterministic Polynomial (NP) problem in the tobacco company. In general, the delivery pattern with "one-level dispatching" and "two-level delivering" is used to simplify the tobacco logistics procedure. The main problem in the delivery course of the tobacco logistics is to choose an effective vehicle routing. It is a typical nonlinear combined nondeterministic polynomial problem, which cannot be solved by current heuristic searching algorithms because of too many variables leading to large nonlinear floating point computations.

Fortunately, when performing the optimizations about complex combined nondeterministic polynomial problem, the optimal solution can be obtained more quickly and effectively by using the population-based algorithms than the algorithms that consider only a single search direction at a time. Particle Swarm Optimization (PSO), presented by J. Kennedy and R.C. Eberhart in [1,2], is a populationbased global robust searching method by manipulating the insect swarm behaviors, where each individual is treated as single particle flying around in a multidimensional search space. R.C. Eberhart and Y. Shi in [3] point out that the behavior of a system optimizing by PSO algorithm is more competitive than that of genetic algorithms. Currently, the PSO with simple evolution procedure is extensively applied in many fields, such as parameter optimizing, pattern classification and routes optimization.
However, the PSO algorithm has some intrinsic defects in the course of the nonlinear optimization, such as premature convergence, being low efficiency in the later stage and falling into local optima. To improve the comprehensive optimization performance, an evolutionary particle swarm optimization algorithm with the addition of a standard selection mechanism from the evolutionary computation is described in [4]. When designing a niching PSO algorithm, the strong local convergence properties of particles is guaranteed in [5]. J. Liang in [6] proposed three versions of particle swarm optimizers with novel learning strategies to ensure the diversity of the swarm. Z. Liping in [7] proposed a new method by introducing the inertia weight employing random number uniformly distributed in $[0,1]$. For the tobacco logistics, the quantum-behaved particle swarm optimization is used to tackle the combined nondeterministic polynomial problem and the optimal distributing route is given in $[8,9]$.

With the combined nondeterministic polynomial delivery problem in tobacco logistics, we propose an improved particle swarm optimization method by introducing the cross-over operation and the mutation operation to the particles in this paper. The content is organized as follows: the tobacco logistics problem is described and its mathematic model is built in Section II. The evolution principle of the PSO is introduced and an Improved Particle Swarm Optimization (IPSO) by using cross-over operation and mutation operation is presented in Section III. In Section IV, an example about tobacco logistics is used to demonstrate the effectiveness to design the vehicle routes, and Section $\mathrm{V}$ is the conclusions.

\section{MOdEl OF TOBACCO LOGISTICS PROBLEM}

\section{A. Problem Description}

Because of large quantity demanded, high frequentness, extensive scope and scattered distribution, the delivery procedure of tobacco logistics in China contains the following two regular modes, "one-level dispatching" and "two-level delivering". The former is suitable for the small scope around the city, and the later is usually used to the distribution of the county which is in some inconvenience communications or circuitous areas. In this way, how to choose the effective vehicle routes to finish the distribution assignment quickly and punctually is the major difficulty point need to be solved. The delivery procedure of tobacco logistics is given in Figure 1. 


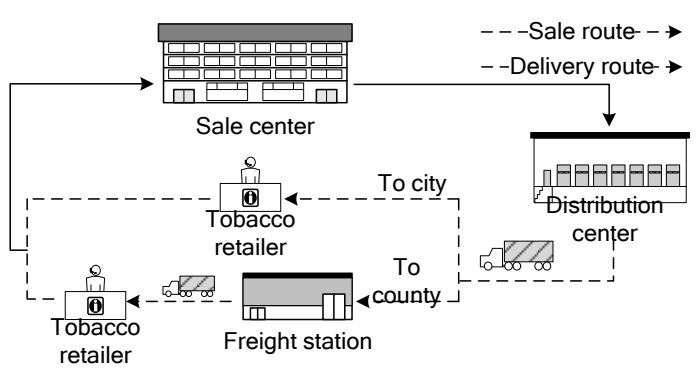

Figure 1. Delivery procedure of tobacco logistics.

\section{B. The Vehicle Route Model}

To build mathematic model of vehicle route in tobacco logistics, we make some reasonable assumptions as follow:

- There is only one available distribution center, where all the vehicles start from and return to after accomplishing the assignment.

- For arbitrary retailer, the tobacco distribution is achieved by a single vehicle.

- The average demand of each tobacco retailer is known and fixed.

- The totality of all the vehicles, which are the same type, is fixed in the distribution center.

- The external factors, such as the bad weather, the blocking traffic, and faulted vehicle, are neglected.

Before conveniently describing the type of the tobacco distributing vehicle, we define that

$$
\Theta_{z}^{x}=\left\{\begin{array}{l}
1, \text { vehicle } z \text { distributes retailer } x \\
0, \text { the others. }
\end{array}\right.
$$

$$
\Theta_{z}^{x, y}=\left\{\begin{array}{l}
1, \text { vehicle } z \text { distributes retailer } y \text { from } x \\
0, \text { the others. }
\end{array}\right.
$$

Actuality, the minimum of vehicle distributing route in a single dispatching period is taken as the optimization objective. Then the distributing problem can be described and modeled as follows. The vehicles number is $m$ in distribution center. The according load is expressed as $\boldsymbol{Q}=\left[Q_{1}, Q_{2}, \cdots, Q_{m}\right]^{\mathrm{T}}$ respectively. In single dispatching period, the demand of tobacco retailer is expressed as $\boldsymbol{D}=\left[D_{1}, D_{2}, \cdots, D_{n}\right]^{\mathrm{T}}$, which satisfies $\sum D_{i} \leq \sum Q_{k}$. For the arbitrary dispatching process, if any vehicle $m_{r}$ needs to make the services for tobacco retailers $n_{r}$, then $\sum D_{i} \leq Q_{m_{r}}$. We note the distribution center as $W_{o}$, and the tobacco retailers is $W_{1}, W_{2}, \ldots, W_{n}$ successively. The haul distance from the retailers $i$ to the retailers $j$ is $d_{i j}$, accordingly the haul distance from distribution center to the retailers is $d_{o i}(i, j=1,2, \cdots, n)$. The set $R_{k}$ denotes the $k$-th route of the vehicle, whose elements express the sort of the retailer $j$ is $i$ in the route $k$. And the expression $e_{k 0}=0$ means the distribution center. Then the mathematic model of the vehicle route optimization in a period for the tobacco delivery can be described as

$$
\begin{aligned}
& \min _{W} \boldsymbol{J}=\boldsymbol{\omega}\left(\sum_{r=1}^{m}\left[\sum_{i=1}^{n_{r}} d_{e_{r(t-1)} e_{r}}+d_{e_{m_{r} e_{r 0}}} \operatorname{sign}\left(n_{r}\right)\right]\right) \\
& \text { s.t. }\left\{\begin{array}{l}
\sum_{i=1}^{n} D_{i} \leq \sum_{k=1}^{m} Q_{k}, \quad \sum_{i=1}^{n_{r}} D_{i} \leq Q_{m_{r}} . \\
\sum_{z} \Theta_{z}^{x}=1 . \\
\sum_{x} \Theta_{z}^{x, y}=\Theta_{z}^{y}, \quad \sum_{y} \Theta_{z}^{x, y}=\Theta_{z}^{x}, \quad \forall z . \\
\Theta_{z}^{x, y}, \Theta_{z}^{x}, \Theta_{z}^{y}=1 \text { or } 0 . \\
x, y=1, \ldots, n . \quad z=1, \ldots, m .
\end{array}\right.
\end{aligned}
$$

Where $\omega$ is the cost function related to vehicle route, and the optimal $\boldsymbol{J}$ implies the minimum of the distributing cost. If $n_{r} \geq 1$, then $\operatorname{sign}\left(n_{r}\right)=1$. Otherwise, $\operatorname{sign}\left(n_{r}\right)=0$.

\section{IMPROVED PARTICLE SWARM OPTIMIZATION}

\section{A. Particle Swarm Optimization}

Particle Swarm Optimization has been proven to be very effective for many optimization problems. Like other evolutionary technique, such as genetic algorithms (GA) or ant colony optimization (ACO), the particles in the swarm are correspondingly associated with their own fitness about the optimization problem through the position and velocity of the particle in a multidimensional search space. Instead of traditional evolutionary operators such as mutation or crossover, PSO is inspired by the advantage of cooperative and social behavior seen in swarm intelligence. In addition, the particles in the swarm share their flying information together and point out what points of the search space are promising. Then the individual particles learn from their neighbors as well as themselves to fly to the promising particle until now.

First of all, some terminologies need to be defined for the evolutionary model building. If the dimension search space of the particle is $D$, PSO algorithm is attempting to find an optimal location with respect to a fitness function $f$. The population of particles in the swarm is denoted with $G$, which form the multidimensional space

$$
\boldsymbol{X}=\left\{\boldsymbol{X}_{1}, \boldsymbol{X}_{2}, \cdots, \boldsymbol{X}_{G}\right\}
$$

And the according position and velocity of the $i$-th particle can be expressed as

$$
\begin{gathered}
\boldsymbol{X}_{i}=\left(X_{i 1}, X_{i 2}, \cdots, X_{i D}\right)^{\mathrm{T}} . \\
\boldsymbol{V}_{i}=\left(V_{i 1}, V_{i 2}, \cdots, V_{i D}\right)^{\mathrm{T}} .
\end{gathered}
$$

We define the best previous position of a particle (local best) as

$$
\boldsymbol{P}_{i}=\left(P_{i 1}, P_{i 2}, \cdots, P_{i D}\right)^{\mathrm{T}}
$$

and define the best position of a particle (global best) as 


$$
\boldsymbol{P}_{g}=\left(P_{g 1}, P_{g 2}, \cdots, P_{g D}\right)^{\mathrm{T}},
$$

such that $f$ is a decline function about the local best $\boldsymbol{P}_{i}$ and the global best $\boldsymbol{P}_{g}$.

Using the above defined notations, each particle $\boldsymbol{X}_{i}$ in the swarm moves with updated position and velocity according to the following equations.

$$
\begin{gathered}
V_{i d}^{k+1}=w^{k} V_{i d}^{k}+\left[\begin{array}{ll}
c_{1} r_{1} & c_{2} r_{2}
\end{array}\right]\left[\begin{array}{ll}
P_{i d}^{k}-X_{i d}^{k} & P_{g d}^{k}-X_{i d}^{k}
\end{array}\right]^{\mathrm{T}} \\
X_{i d}^{k+1}=X_{i d}^{k}+V_{i d}^{k+1}
\end{gathered}
$$

where $d=1,2, \cdots, D, i=1,2, \cdots, G, k$ is the generation number in the evolution, $w^{k}$ is the inertia weight, $c_{1}$ and $c_{2}$ are two positive constants, $r_{1}$ and $r_{2}$ are uniformly distributed random number in $[0,1]$. The inertia weight $w^{k}$ determines the influence of the previous velocity on the current velocity.

All the positions and velocities of the particles in the swarm are initialized to the random values within some boundaries at first. At every generation, the fitness for the particles is evaluated and the positions and velocities are updated. If some criterion set in advance is met, such as the number of iterations or stagnation fitness, the evolutionary will be stopped.

\section{B. Improved Particle Swarm Optimization}

The major shortcoming of the PSO algorithm is that enough knowledge of the search space is needed to efficiently attract the swarm to the optimal particle and adjust the balance of exploration vs. exploitation. According to the equation (10) and (11), the convergence rate of the particle swarm can be adjusted by many factors, including the number of particles, neighbor topology and parameters selection. All these measures do not change the optimization structure of the particle swarm. The clustering feature of the particles has decided that the algorithm tends to fall into premature convergence, which is highly undesirable in real optimization.

For the tobacco logistics problem with high dimension and nonlinearity optimization, the variety of the population will decrease when the local best $\boldsymbol{P}_{i d}^{k}$ is be close to the global best $\boldsymbol{P}_{g d}^{k}$. Then the particle swarm is probable to be premature convergence and be lost into local best.

For that reason, we introduce the cross-over operation and the mutation operation ${ }^{[10,11]}$ to the particles. In every generation, the new population is produced by the crossover and mutation of the former particles. The evolution procedure is as follows:

- According to the equation (10) and (11), obtain the positions and velocities of the particles.

- Evaluate the fitness for the particles, and sort the fitness by ascending order.

- Divide the population into a lot of family equally. Every family illustrates some class of particles.

- Choose a particle from each family to form the new child family.
- The cross-over operation and mutation operation is performed in the new child family, which generates the new child population.

- Because the number of the population is much more than the one before evolution, choose the particles with large fitness to form the new population from the child population and father population.

With regard to the particles in the child family, the positions and velocities information is made cross-over operation and mutation operation randomly. If the particles in the child family denote $\boldsymbol{x}$, the positions and velocities of the next generation is updated by

$$
\begin{aligned}
& \operatorname{Child}_{1}(\boldsymbol{x})=\boldsymbol{p} \times \operatorname{Parent}_{1}(\boldsymbol{x})+(1-\boldsymbol{p}) \times \operatorname{Parent}_{2}(\boldsymbol{x}) \\
& \operatorname{Child}_{2}(\boldsymbol{x})=\boldsymbol{p} \times \operatorname{Parent}_{2}(\boldsymbol{x})+(1-\boldsymbol{p}) \times \operatorname{Parent}_{1}(\boldsymbol{x}) \\
& \text { Child }_{1}(\boldsymbol{v})=\frac{\operatorname{Parent}_{1}(\boldsymbol{v})+\operatorname{Parent}_{2}(\boldsymbol{v})}{\left|\operatorname{Parent}_{1}(\boldsymbol{v})+\operatorname{Parent}_{2}(\boldsymbol{v})\right|} \times \mid \text { Parent }_{1}(\boldsymbol{v}) \mid \\
& \operatorname{Child}_{2}(\boldsymbol{v})=\frac{\operatorname{Parent}_{1}(\boldsymbol{v})+\operatorname{Parent}_{2}(\boldsymbol{v})}{\left|\operatorname{Parent}_{1}(\boldsymbol{v})+\operatorname{Parent}_{2}(\boldsymbol{v})\right|} \times\left|\operatorname{Parent}_{2}(\boldsymbol{v})\right|
\end{aligned}
$$

where the symbol Child and Parent are stand for the child particle and father particle respectively. The parameter $\boldsymbol{p}$ is random numbers uniformly distributed in $[0,1]$.

Through introducing the cross-over operation and the mutation operation to the particles, the child particles can inherit the advantages of the father particles, which enhance the region searching capability of the particles in theory. At the same time, the mutation operation between the particles made the Hemming distance of the father particles be controllable, which avoids the inbreeding coefficient on particles and is fit for keeping the diversity of the population.

\section{NUMERICAL SIMULATION AND VALIDATION}

To investigate the benefits of the above mentioned method about the improved particle swarm optimization, we choose a city with 9 fixed retailer points. The network constructed by the distributing route is shown in Figure 2. There are probable $3.63 \times 10^{6}$ distributing routes in the case of all the retailer points finishing delivery. However, the number of the distributing routes is raised rapidly when the scope of service expands or the retailer points increases. These factors may lead much more workload to the distribution center directly. Therefore, it is particularly important to design an effective distributing route in a short time. The requirement of each retailer is shown in Table I.

TABLE I. THE REQUIREMENT OF EACH RETAILER

\begin{tabular}{|c|c|c|c|c|c|}
\hline Retailer & $\mathbf{W}_{\mathbf{1}}$ & $\mathbf{W}_{\mathbf{2}}$ & $\mathbf{W}_{\mathbf{3}}$ & $\mathbf{W}_{\mathbf{4}}$ & $\mathbf{W}_{\mathbf{5}}$ \\
\hline Requirement & 62 & 23 & 32 & 28 & 42 \\
\hline Retailer & $\mathbf{W}_{\mathbf{6}}$ & $\mathbf{W}_{\mathbf{7}}$ & $\mathbf{W}_{\mathbf{8}}$ & $\mathbf{W}_{\mathbf{9}}$ & \\
\hline Requirement & 53 & 19 & 12 & 37 & \\
\hline
\end{tabular}




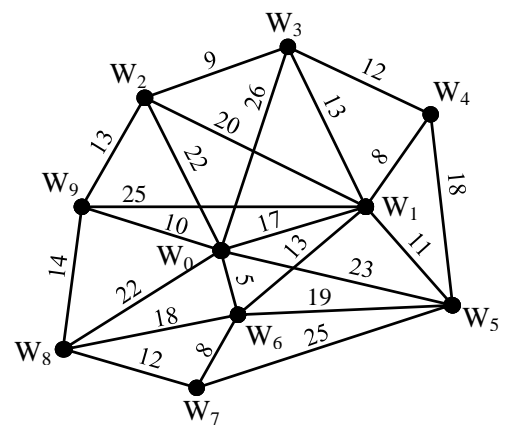

Figure 2. Distributing network of tobacco logistics.

The population of the swarm is initialized as 50. The fitness of each particle is set as 10000. The positive constants is defined as the same value, $c_{1}=c_{2}=1.49445$. Then the evolutionary course is stopped until the generation number runs up to 500. In the improved particle swarm optimization, the particles are divided into three families with the same numbers.

In order to validate the effectiveness of the improved particle swarm optimization, the following two methods are compared.

- PSO: According to the equation (10) (11), the positions and velocities of the particles can be updated.

- IPSO: According to the equation (10) (15), the positions and velocities of the particles can be updated.

Then the local best fitness and the global best fitness will be obtained after a little computation. The convergence curves of the fitness about the above two methods is shown in Figure 3.

According to Figure 3, we can obtain that the best fitness of the IPSO is much smaller than the one of the PSO, which means the IPSO can solve the combined nondeterministic polynomial problem in tobacco logistics better. In the beginning, the fitness of PSO is declined to the best value much faster than that of IPSO. But it falls into the premature convergence at the point $f=120$. Through the cross-over operation and mutation operation of the particles, the IPSO is converged to the point $f=115$. The according best distributing route is shown in Table II.

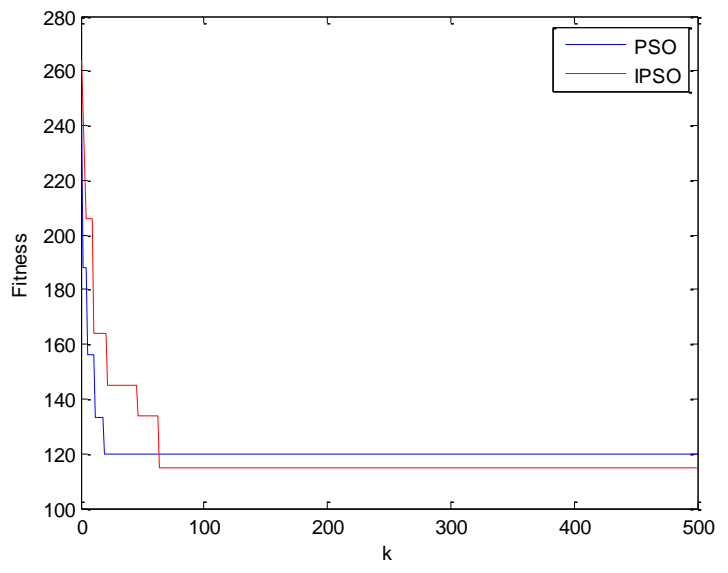

Figure 3. The convergence curves of the fitness.
TABLE II. The Best Distributing Route

\begin{tabular}{|c|c|c|}
\hline Algorithm & The best distributing route & The distance \\
\hline PSO & $\begin{array}{r}\mathrm{W}_{0} \rightarrow \mathrm{W}_{7} \rightarrow \mathrm{W}_{8} \rightarrow \mathrm{W}_{9} \rightarrow \mathrm{W}_{2} \rightarrow \mathrm{W}_{3} \\
\rightarrow \mathrm{W}_{4} \rightarrow \mathrm{W}_{5} \rightarrow \mathrm{W}_{1} \rightarrow \mathrm{W}_{6} \rightarrow \mathrm{W}_{0}\end{array}$ & 120 \\
\hline IPSO & $\begin{array}{r}\mathrm{W}_{0} \rightarrow \mathrm{W}_{6} \rightarrow \mathrm{W}_{7} \rightarrow \mathrm{W}_{8} \rightarrow \mathrm{W}_{9} \rightarrow \mathrm{W}_{2} \\
\rightarrow \mathrm{W}_{3} \rightarrow \mathrm{W}_{4} \rightarrow \mathrm{W}_{1} \rightarrow \mathrm{W}_{5} \rightarrow \mathrm{W}_{0}\end{array}$ & 115 \\
\hline \multicolumn{2}{|c}{}
\end{tabular}

\section{CONCLUSIONS}

For the distributing route problem in tobacco logistics considered in this paper, an evolutionary method by introducing the cross-over operation and the mutation operation to the particles in the swarm is presented. Based on the particles updating principle of the positions and velocities in the standard PSO, the grouping scheme is used to divide the particles into many families with the same number. After the particle finishing cross-over operation and mutation operation, the diversity and performance of the population is increasing largely. At last, an example with nine distributing points is used to validate the effectiveness of the improved particle swarm optimization.

\section{REFERENCES}

[1] J. Kennedy and R.C. Eberhart, "Particle swarm optimization," Proceedings of IEEE International Conference on Neural Networks, IEEE Service Center, Piscataway, Nov. 1995, pp. 1942-1948, doi: 10.1109/ICNN. 1995.488968.

[2] J. Kennedy, "The particle swarm social: adaptation of knowledge," Proceedings of IEEE International Conference on Evolutionary Computation, IEEE Service Center, Piscataway, Apr. 1997, pp 303-308, doi: 10.1109/ICEC. 1997.592326.

[3] R.C. Eberhart and Y. Shi, "Comparison between genetic algorithms and particle swarm optimization," Evolutionary Programming VII: Proceedings of the Seventh Annual Conference on Evolutionary Programming, Springer Berlin Heidelberg, Mar. 1998, pp. 611-617, doi: 10.1007/BFb0040812.

[4] P.J. Angeline, "Using selection to improve particle swarm optimization," IEEE International Conference on Evolutionary Computation, IEEE Service Center, Piscataway, 1998, pp. 84-89, doi: 10.1109/ICEC. 1998.699327.

[5] F.Van den Bergh and A.P. Engelbrecht, "A new locally convergent particle swarm optimizer," Proceedings of the IEEE international Conference on Systems, Man and Cybernetics (SMC 02), Oct. 2002, vol. 3, pp. 96-101, doi:10.1109/ICAMC.2002.1176018.

[6] J.J. Liang, A.K. Qin, P.N. Suganthan and S. Baskar, "Particle swarm optimization algorithms with novel learning strategies," Proceedings of the IEEE international Conference on Systems, Man and Cybernetics (SMC 04), Oct. 2004, vol. 3, pp. 3659-3664, doi:10.1109/ICAMC.2004.1400911

[7] Z. Liping, Y. Huangjun and H. Shangxu, "A new approach to improve particle swarm optimization," Genetic and Evolutionary Computation Conference (GECCO 03), July 2003, pp.134-139, doi: 10.1007/3-540-45105-6_12.

[8] Zhao Li, "Strategy for the optimization of tobacco distribution based on QPSO," Logistics technology, vol. 222, Aug. 2010, pp. 86-88, doi:10.3969/j.issn.1005-152X.2010.15.028.

[9] Zhao Li and Gao Lijun, "Modified GQPSO for tobacco delivery scheme with mixed optimization," Logistics Sci-Tech, vol. 11, Nov. 2010, pp. 63-66.

[10] J. McCall, "Genetic algorithms for modelling and optimisation," Journal of Computional and Applied Mathematics, vol. 184, issue 1, Dec. 2005, pp. 205-222, doi:10.1016/j.cam.2004.07.034.

[11] K. Abdullah, W.C. David and A.E. Smith, "Multi-objective optimization using genetic algorithms: A tutorial," Reliability Engineering \& System Safety, vol. 91, issue 9, Sep. 2006, pp. 9921007, doi: 10.1016/j.ress.2005.11.018 